\title{
Die Melorheostose - eine Differenzialdiagnose maligner Knochentumoren im jungen Erwachsenenalter
}

\section{Melorheostosis - a benign entity mimicking malignant bone tumor in young adults}

\section{Einleitung}

Die Melorheostose (MRH, Syn.: Kerzenwachskrankheit) ist eine sporadische kongenitale Erkrankung aus der Gruppe der sklerosierenden Knochendysplasien, die zu fokal überschüssigen Knochenformationen führt. Die geschätzte Prävalenz liegt bei 0,9 pro 1000000 Personen (Wynne-Davies and Gormley, 1985), Männer und Frauen sind gleich häufig betroffen. Eine Assoziation mit weiteren sklerosierenden Knochendysplasien (z. B. Osteopoikilose) und dem Buschke-Ollendorff-Syndrom wurde beschrieben, die genaue Pathogenese ist jedoch ungeklärt. Obgleich es sich um eine benigne Erkrankung handelt, können ein extensives und gelenksüberschreitendes Wachstum sowie die Infiltration umliegender Muskeln und Sehnen zu massiven Einschränkungen der Patienten führen. Typische Symptome umfassen Schmerzen, Knochendeformierung und Bewegungseinschränkung, Muskelschwäche und -atrophien sowie Taubheitsgefühle (Judkiewicz et al. 2001; Smith et al. 2017). Veränderungen des darüber liegenden Bindegewebes und der Haut, wie beispielsweise Hyperpigmentierung, subkutane Fibrose und vaskuläre Malformationen, sind häufig.

Die Erkrankung verläuft langsam-progressiv.
Die Diagnosestellung erfolgt radiologisch. Klassische Befunde sind multiple peri- und endosteale Hyperostosen mit segmentalem und unilateralem (monomelischem) Verteilungsmuster (Smith et al. 2017). Prädilektionsstelle sind die Diaphysen der Röhrenknochen, die untere Extremität ist am häufigsten betroffen. Es werden 4 morphologische Subtypen unterschieden (klassische Kerzenwachsform, Osteopathia-striata-ähnliche, Myositisossificans-ähnliche, Osteom-ähnliche Form) (Freyschmidt 2001).

- Tab. 1 Klinische und radiologische Charakteristiken der Myositis-ossificans-like Melorheostose und ihrer wichtigsten Differenzialdiagnosen.

\begin{tabular}{|c|c|c|c|}
\hline & Myositis ossificans & parosteales Osteosarkom & Myositis-ossificans-like Melorheostose \\
\hline Entität & $\begin{array}{l}\text { benigne fibroossäre Läsion, } \\
\text { Pseudotumor }\end{array}$ & Low-grade-Knochensarkom & sklerosierende Knochendysplasie \\
\hline Manifestationsalter & 2.-3. Dekade & 3. Dekade & 2.-3. Dekade \\
\hline Inzidenz & $\begin{array}{l}<1 / 1000000 \text { Personen, } \\
\mathrm{m}>\mathrm{f}\end{array}$ & $\begin{array}{l}4 \% \text { aller Osteosarkome, } \\
\mathrm{f}>\mathrm{m}\end{array}$ & $\begin{array}{l}0,9 / 1000000 \text { Personen, } \\
\mathrm{m}=\mathrm{f}\end{array}$ \\
\hline Therapie & - symptomorientiert & $\begin{array}{l}\text { " weite Exzision (>1 cm chirurgische Ränder) } \\
\text { " neoadjuvante Chemotherapie in } \\
\text { ausgewählten Fällen }\end{array}$ & " symptomorientiert \\
\hline Prognose & $\begin{array}{l}\text { keine Einschränkung } \\
\text { der Lebenserwartung }\end{array}$ & 5-Jahres-Überleben 86-91\% & $\begin{array}{l}\text { keine Einschränkung } \\
\text { der Lebenserwartung }\end{array}$ \\
\hline Diagnostik & Röntgen, native CT, MRT & Histopathologie & Röntgen, native CT, MRT \\
\hline Krankheitsverlauf & $\begin{array}{l}\text { - initial rasches Wachstum } \\
\text { - selbstlimitierend } \\
\text { - oft spontane Rückbildung }\end{array}$ & $\begin{array}{l}\text { - langsames Wachstum (Jahre) } \\
\text { - High-grade-Transformation (16-43\%) }\end{array}$ & - sehr langsames Wachstum \\
\hline Lokalisation & $\begin{array}{l}\text { - Skelettmuskulatur (Vastus- } \\
\text { Gruppe) } \\
\text { " untere > obere Extremität }\end{array}$ & $\begin{array}{l}\text { - Metaphyse von Röhrenknochen (poste- } \\
\text { riorer Aspekt des distalen Femur 70\%) } \\
\text { " periosteal } \\
\text { - untere > obere Extremität }\end{array}$ & $\begin{array}{l}\text { - peripheres Skelett } \\
\text { - juxtakortikal, periartikulär } \\
\text { - untere > obere Extremität }\end{array}$ \\
\hline Verteilungsmuster & solitär & solitär (Metastasen sind selten) & multipel, segmentale Verteilung \\
\hline Mineralisation & $\begin{array}{l}\text { - graduell } \\
\text { - zonal (peripher > zentral) } \\
\text { - reifer, lamellärer Knochen }\end{array}$ & $\begin{array}{l}\text { - irregulär } \\
\text { - revers-zonal (zentral > peripher) } \\
\text { - reifer, trabekulärer Knochen }\end{array}$ & $\begin{array}{l}\text { - irregulär } \\
\text { - Osteoid-ähnlicher, dichter Knochen }\end{array}$ \\
\hline Periostreaktion & keine („string sign“) & $\begin{array}{l}\text { - kortikale Verdickung oder Erosion } \\
\text { - minimale Periostreaktion } \\
\text { " „Cleavage plane“ (65\%) }\end{array}$ & - variabel \\
\hline Markrauminfiltration & keine & ja $(22-58 \%)$ & keine \\
\hline Kontrastmittelaufnahme & kräftig, zentral betont & heterogen, Weichgewebskomponenten & heterogen, Weichgewebskomponenten \\
\hline
\end{tabular}




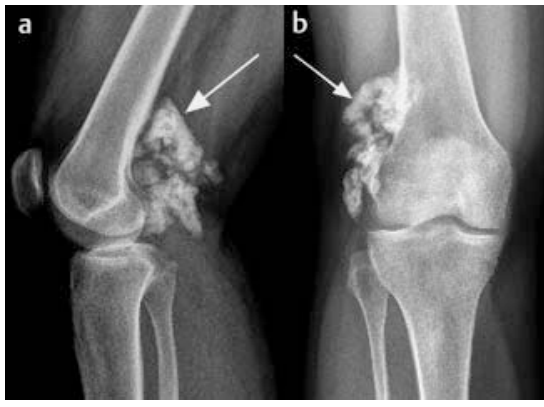

- Abb. 1 Konventionelle Röntgenaufnahme des rechten Knies einer 39-jährigen Patientin mit Myositis-ossificansähnlicher Melorheostose. Laterale a und a. p. b Projektion zeigen eine wolkenartig kalzifizierte Expansion (Pfeile) in dorsalen Oberschenkelweichteilen mit Kontakt zum distalen Femur.

\section{Fallbeschreibung}

Eine zuvor gesunde 39-jährige Patientin stellte sich in der orthopädischen Ambulanz unserer Klinik aufgrund belastungsabhängiger Schmerzen und einer Bewegungseinschränkung im rechten Kniegelenk vor. Anamnestisch lag kein Trauma vor. Klinisch fiel eine tastbare, druckdolente Verhärtung im Bereich der rechten Fossa poplitea auf.

Im konventionellen Röntgen des rechten Knies ( $\mathbf{A b b . 1 )}$ zeigte sich eine wolkenartig kalzifizierte bzw. ossifizierte Expansion in den distalen dorsalen Oberschenkelweichteilen mit kurzstreckigem Kontakt zum distalen Femur.

Konkordant hierzu zeigte die native Computertomografie (CT) ( $\mathbf{A} \mathbf{b b} \mathbf{b}$. 2a) eine solide, maximal 7,2 cm große, juxtakortikale Expansion mit irregulärer Mineralisierung und solider Periostreaktion des distalen Femurs. In der weiterführenden Magnetresonanztomografie (MRT) ( $\mathbf{A} \mathbf{b} \mathbf{b} . \mathbf{2} \mathbf{b}-\mathbf{d}$ ) fand sich ein moderates Enhancement im nichtmineralisierten Läsionsanteil. Eine endosteale Verdickung sowie ein minimales Knochenmarködem des Femurs lagen vor, jedoch keine Kontrastmittelaufnahme im Sinne einer Knochenmarkinfiltration.

Nebenbefundlich fielen in der 3-phasigen MBq-99m-Tc-DPD-PET-CT 2 intraossäre Läsionen mit gesteigertem Tracer-Uptake der Fibuladiaphyse und des Calcaneus auf. Die Läsionen konnten mittels MRT und CT verifiziert werden ( $\bullet$ Abb. $\mathbf{3}$ ). Als begleitende Weichgewebsveränderungen zeigten sich auf den rechten Unterschenkel be-
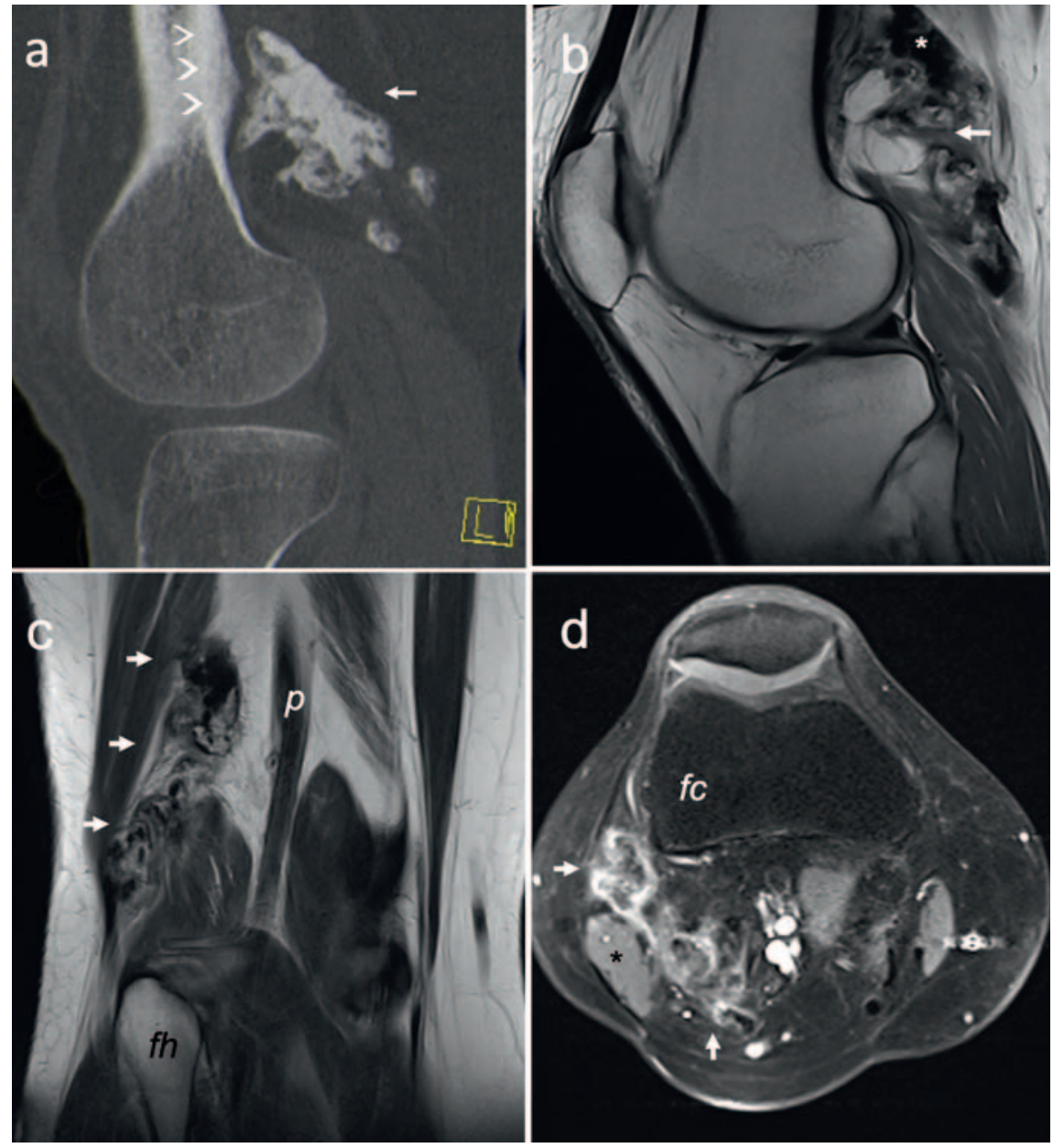

- Abb.2 CT- und MRT-Untersuchung des rechten Knies einer 39-jährigen Patientin mit Myositis-ossificans-ähnlicher Melorheostose. CT des rechten Knies in sagittaler Schicht a zeigt eine irregulär und Osteoid-ähnlich kalzifizierte Expansion mit 7,2 cm maximalem Durchmesser in den dorsalen Oberschenkelweichteilen (Pfeil). Es besteht eine Nahebeziehung zur dorsalen Femurmetadiaphyse mit breiter Kortikalisreaktion, jedoch mit fehlender Verbindung zur Kompakta (Pfeilköpfe). Die sagittale native T1w b verdeutlicht die Heterogenität der Läsion mit kalzifizierten (Stern) und nichtkalzifizierten (Pfeil) Anteilen sowie die Nähe zum Caput laterale des M. gastrocnemius. In der koronalen nativen T1w c zeigt sich ein breitflächiger Kontakt zum M. biceps femoris und M. plantaris (Pfeile), teils mit unscharfer Begrenzung der Expansion. Kein direkter Kontakt zu A. poplitea (p) und zum Fibulaköpfchen (fh). Die axiale fettgesättigte T1w nach Kontrastmittelgabe d demonstriert eine heterogene Kontrastmittelaufnahme der Expansion (Pfeile) sowie deren Nahebeziehung zum M. biceps femoris (Stern) und lateralen Femurkondyl (fc).

schränkte, subkutane venöse Malformationen.

Initial lenkten Lokalisation und Morphologie der femoralen Expansion den Verdacht auf ein parosteales Osteosarkom. Differenzialdiagnostisch wurden ein kalzifiziertes Hämatom und eine Myositisossificans-like MRH diskutiert. Im interdisziplinären Konsens erfolgte die endgültige Diagnosesicherung mittels Inzisionsbiopsie. Histologisch ergab sich Weichgewebe und verbreitertes Kortikalis-artiges Knochengewebe, vereinbar mit einer MRH.
Auf Wunsch der Patientin wurde eine chirurgische Exzision der femoralen Läsion vorgenommen. Bei klinischer Symptomfreiheit ergab das 4 Monate später angefertigte Kontrollröntgen keinen Anhalt für ein Lokalrezidiv.

\section{Diskussion}

Die klassische Kerzenwachsform der MRH macht etwa ein Fünftel der Fälle aus und zeigt sich im Röntgenbild durch kortikale 

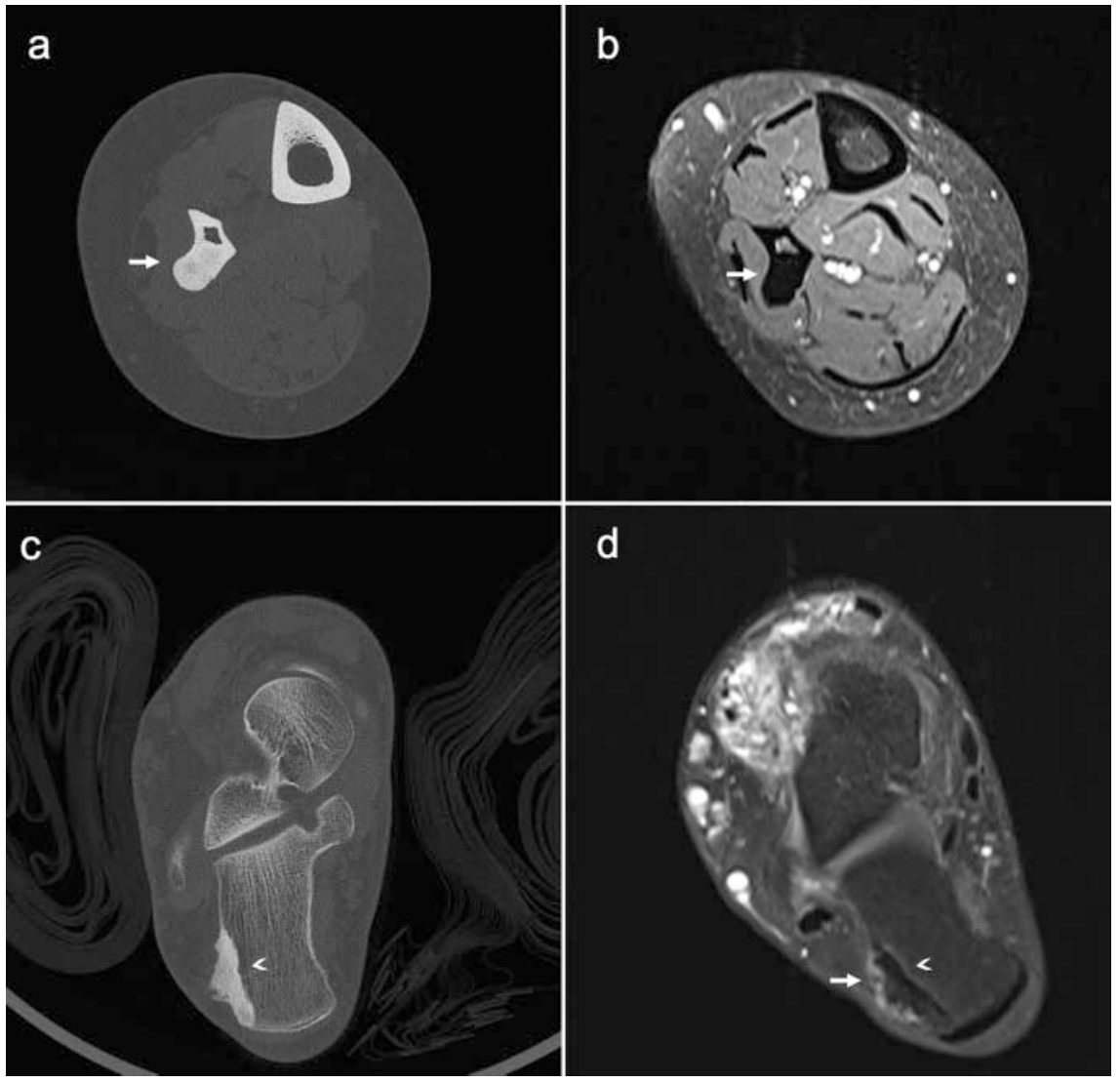

- Abb. 3 Segmentale Verteilung weiterer Melorheostose-Manifestationen am rechten Unterschenkel. Axiale CT (a, c) und MRT (b, d) des rechten Unterschenkels. In der Fibuladiaphyse zeigte sich in CT a und MRT $\mathbf{b}$ in PD-Wichtung eine weitere Melorheostose-Läsion des Kerzenwachstyps. Eine dritte Läsion des Osteom-ähnlichen Typs (c, d) fand sich laterodorsal im Calcaneus (Pfeilspitzen). Beide Läsionen weisen eine end- und periosteale Komponente auf. Die axiale PD-Wichtung $\mathbf{b}$ verdeutlicht das Kortikalis-artige Signalverhalten der exzentrisch gelegenen Läsion im Fibulaschaft, mit end- und periostealer Ausdehnung (Pfeil). In der axialen fettgesättigten T1-Wichtung nach intravenöser Kontrastmittelapplikation kann ein schmales randständiges Enhancement abgegrenzt werden (Pfeil) d.

Verdickungen entlang der Längsachse des Knochens, welche an fließendes Kerzenwachs erinnern (Freyschmidt, 2001).

Charakteristikum der Myositis-like MRH sind kalzifizierte und nichtkalzifizierte, teils palpable Expansionen mit infiltrativem Wachstum (Freyschmidt 2001; Judkiewicz et al. 2001). Diese finden sich gehäuft im periartikulären Weichgewebe, wobei ein Knochenkontakt typischerweise nachweisbar ist.

Das parosteale Osteosarkom und die Myositis ossificans zählen zu den wichtigsten Differenzialdiagnosen der Myositisossificans-ähnlichen MRH. Eine präzise radiologische Unterscheidung ist essenziell, kann jedoch aufgrund der Ähnlichkeit der konventionellen Bildbefunde herausfordernd sein. Die native dünnschichtige CT im Knochen- und Weichteilfenster
Während die Hauptdifferenzialdiagnosen ein zonales (Myositis) und revers-zonales (Osteosarkom) Kalzifizierungsmuster aufweisen, finden sich bei der Myositislike MRH heterogene und irreguläre Mineralisierungen. Die Manifestierung der MRH ist in der Regel polyostotisch, mit mehreren betroffenen Knochen derselben Extremität und nur geringer Größenzunahme im Verlauf. Mehrspeicherungen in Skelettszintigrafie und PET/CT wurden mehrfach beschrieben und sind in erster Linie auf die Läsionsaktivität zurückzuführen (Vyskocil et al. 2015; Jha et al. 2019).

Die Therapie der MRH ist symptomatisch und stark individuell. Gängige Optionen sind die Analgesie, Physiotherapie und seltener die chirurgische Resektion mit dem Ziel der Schmerzfreiheit und Wiederherstellung des vollen Bewegungsumfangs (Smith et al. 2017).

\section{Schlussfolgerung}

Die Myositis-ossificans-ähnliche MRH ist eine seltene benigne Differenzialdiagnose kalzifizierter Weichgewebsexpansionen im jungen Erwachsenenalter. Anders als das parosteale Osteosarkom und die Myositis ossificans liegt die Erkrankung meist multifokal und in segmentaler Verteilung vor. Das Kalzifizierungsmuster der Läsionen ist ein hilfreiches Unterscheidungsmerkmal in der CT. Ein rascher Größenprogress ist untypisch für die MRH. Wir schlussfolgern, dass das klinische Bild, die Patientenhistorie und radiologische Befunde essenziell für die korrekte Diagnosestellung und die Vermeidung weiterer, teils invasiver Diagnostik sind.

\section{Interessenkonflikt}

Die Autorinnen/Autoren geben an, dass kein Interessenkonflikt besteht.

\section{Autorinnen/Autoren}

Ann-Katrin Kaufmann-Bühler, Helmut Schöllnast, Jasminka Igrec(D)

Division of General Radiology, Medical University of Graz, Austria 
Dr. Ann-Katrin Kaufmann-Bühler Division of General Radiology,

Medical University of Graz

Auenbruggerplatz 9

8036 Graz

Austria

Tel.: +43/3 16/38512577

ann.kaufmann-buehler@medunigraz.at
Fortschr Röntgenstr 2022; 194: 416-419

Online-Publikation: 21.12.2021

DOI 10.1055/a-1656-9987

ISSN 1438-9029

(C) 2021. The Author(s).

This is an open access article published by Thieme under the terms of the Creative Commons Attribution-

NonDerivative-NonCommercial License, permitting copying

and reproduction so long as the original work is given

appropriate credit. Contents may not be used for

commecial purposes, or adapted, remixed, transformed or

built upon. (https://creativecommons.org/licenses/by-nc-

nd $/ 4.0 /$ )

\section{(c) (i) $(5$}

